\title{
Efecto de la dieta en el resultado perinatal de la paciente obesa embarazada
}

\author{
Gustavo Gómez Tabares MD*; Julián Delgado G.; Alejandro Agudelo A.**; Hugo Hurtado***
}

\begin{abstract}
RESUMEN: En un estudio prospectivo randomizado se estudian 60 pacientes obesas divididas en grupos de 20 pacientes de acuerdo con el porcentaje de obesidad en: $20-29 \% ; 30-39 \%$ y $30 \%$ de sobrepeso. A la mitad de los pacientes se les instaura dieta similar a la usada en la diabética gestacional. La otra mitad se le administra dieta libre y sirve de control. Se determina peso del RN, morbimortalidad fetal y materna.
\end{abstract}

No se encontró diferencia en el peso del $\mathrm{RN}$; la incidencia de macrosómicos es similar en ambos grupos (13.3\%). No se presentó mortalidad materna ni fetal en ninguno de los grupos. La incidencia de cesáreas por fet os grandes para la edad gestacional en el grupo estudio vs grupo control fue $\mathbf{3 8 . 4 \%}$ vs $\mathbf{5 3 . 8 \%}$ y los antecedentes de familiares de diabetes son más frecuentes a medida que aumenta la obesidad. En los tres grupos de obesidad del grupo estudio se encontró correlación entre el peso del RN y la edad gestacional, contrario a lo encontrado con el grupo control. Se concluye que aunque en este estudio no hay diferencia en la morbimortalidad materna-fetal, ni en el peso del RN, con relación a recibir o no dieta, ésta es benéfica en la regulación del metabolismo energético fetal que lo lleva a adquirir una relación normal del crecimiento fetal.

PALABRAS CLAVES: Obesa, embarazo, sobrepeso, dieta, morbimortalidad.

SUMMARY: We studied prospectively 60 obese patients divided in 3 group of 20 patients each with overweigth of $20-29 \%$; $30-39 \%$ and $3 \mathbf{4 0 \%}$ respectively. Half of the patients were under diet for gestational diabetes and the other half served as control. We determined newborn weigth, and morbimortality fetal and maternal. There were no mortality either fetal or maternal. There were not difference in the newborn weight in both groups. The macrosomics were $13.3 \%$ in both groups. The incidence o cesarean section because large babies for gestational age was $38.4 \%$ vs $53.8 \%$ in the study vs control group. In the three obesity groups of diet patients there were positive correlation between the newborn weigth and the gestational age, contrary to in the control group happen. We concluded that instead there are not diferences in newborn weigth, and maternal and fetal morbimortality the correlation of fetal weigth and gestational age is derived of a regulation in the fetal energetic metabolism that will be beneficious and become in a good relationship in the fetal growth.

KEY WORDS: Obese, pregnancy, overweigth, diet, morbimortality.

Se define como obesidad, cuando la cantidad de tejido adiposo es lo suficientemente alta para producir alteraciones bioquímicas y fisiológicas que acorten la expectativa de vida. Ponderalmente ocurre cuando hay un $20 \%$ o más del peso ideal para la talla (1).

Constituye el 10-12\% de la población de los Estados Unidos, con una tendencia a aumentar con la edad y el desarrollo socio-económico (2).

La definición de obesidad materna es menos unificada y se han utilizado en diversos trabajos diferentes criterios para ello (3-5).

En un trabajo previo definimos obesidad materna cuando el peso relacionado con la talla era superior a un incremento desde el $20 \%$ sobre el peso ideal, para la edad del embarazo (6).

* Profesor Titular. Departamento de Obstetricia y Ginecología. Universidad del Valle.

** Ginecólogo Asistencial. Hospital Universitario del Valle.

*** Profesor Titular. Departamento de Medicina Social. Universidad del Valle.
Aunque los hallazgos en la búsqueda de la relación causa efecto son inconsistentes, la obesidad, incrementa el riesgo para varias enfermedades como: diabetes mellitus, enfermedad cardiovascular, transtornos endocrinos y cáncer, entre otras.

Entre las alteraciones que genera la obesidad, una de las más importantes es la hiperinsulinemia, secundaria en parte a una disminución del número de receptores a la insulina.

La obesidad por lo tanto es un estado de alto riesgo para diabetes, y para muchos es considerado un estado prediabético (7-8).

Se diseñó este estudio prospectivo de casos y controles con el fin de comparar el efecto de la dieta en pacientes obesas, con relación al resultado perinatal y morbilidad materna.

\section{Pacientes y métodos}

Se incluyeron 60 pacientes obesas que consultaron a la consulta de Endocrinología Obstétrica de la clínica de Embarazo de Alto Riesgo en el Hospital Universitario del Valle. 
Se clasificaron de acuerdo con el porcentaje de sobrepeso con relación a lo ideal, en: obesidad leve (20$29 \%$ ); moderada (30-39\%) y severa (40\% o más).

Cada grupo estuvo constituido por 10 pacientes obesas con dieta y 10 obesas sin dieta como control, para un total de 30 casos y 30 controles.

El porcentaje de sobrepeso determinó calculando según la tabla de peso ideal de acuerdo con la contextura grácil, moderada o fuerte, relacionada con la talla, sumándole 1.1 kilo por cada mes de embarazo (6).

Previo a incluir la paciente en el estudio se realizó, al momento del ingreso, un test con carga de 50 gramos de glucosa y durante la semana 28 de embarazo una curva de tolerancia oral a la glucosa con carga de 100 gramos de glucosa, de acuerdo con el protocolo previamente descrito (9). Si ambos test eran negativos para diabetes, es decir, glicemia en ayunas menor de $90 \mathrm{mgs} / \mathrm{dl}$. y valores post carga $140 \mathrm{mgs} / \mathrm{dl}$ y ningún valor anormal en la curva, respectivamente, se incluyeron aleatoriamente en dos grupos: uno de control con dieta libre y otro con dieta de 30 calorías por kilo de peso ideal, $50 \%$ de carbohidratos, $30 \%$ de grasas y $20 \%$ de proteínas sin ser menor de 1.600 ni mayor de 2.200 calorías.

Se excluyeron del estudio las pacientes con cualquier alteración en la curva de glicemia, las que evidentemente no cumplieron con la dieta ordenada y aquellas pacientes que no terminaron el embarazo en el HUV.

Se excluyeron también 8 pacientes que desarrollaron preeclampsia severa o hipertensión arterial severa que estuvieron distribuidas la mitad en el grupo estudio y la otra mitad en el grupo control.

Se evaluó: peso del recién nacido, vía de terminación del embarazo, morbilidad y mortalidad fetal, morbilidad materna, edad gestacional del recién nacido y puntaje de Apgar.

El análisis de los resultados en ambos grupos se hizo por medidas de tendencia central y análisis de significancia estadística por $t$ de Student.

En toda la muestra y en cada grupo en particular, se correlacionó la edad gestacional con el peso del recién nacido.

\section{Resultados}

No hubo diferencias en los grupos en cuanto a: edad promedio, talla, porcentaje de sobrepeso, número de embarazos ni número de controles prenatales.

La mortalidad materna y fetal fue de cero en ambos grupos. El puntaje de Apgar fue igual o mayor de 7 en todos los recién nacidos.

El promedio de peso del recién nacido en las pacientes del grupo control vs el grupo estudio de acuerdo con el porcentaje de sobrepeso se muestra en la figura 2 .

El promedio de peso del RN de los casos vs. los controles en los tres grupos fue así:

En el grupo de $40 \%, 3418554.13$ y 3788454.68 . En el grupo de $30-39 \%$, 3408.5433 .70 y 3608360.24 ; y el grupo de $20-29 \%$, los pesos fueron 3435419.88 y 3169564 .

En el análisis de la muestra total, no hubo diferencia significativa entre el peso del recién nacido del grupo control y el grupo estudio ( $\mathrm{p}=0.01)$.
Cuando se correlacionó semanas de gestación vs. peso del RN en cada grupo, en el grupo control sin dieta no hubo correlación pero si lo fue significativamente en el grupo que recibió dieta.

El porcentaje de mácrosómicos (peso igual o mayor de 4.000 grs., independiente de la edad del embarazo) correspondió al $13.33 \%$ en ambos grupos.

El porcentaje de embarazos prolongados (42 o más semanas de gestación) fue del $9 \%$ en los dos grupos.

La incidencia de cesárea en los dos grupos fue de $43.3 \%$. Se distribuyó en $40.6 \%$ y $46.4 \%$ en el grupo control y el grupo estudio, respectivamente. El $53.8 \%$ de las cesáreas

realizadas en el grupo control fue por fetos macrosómicos y $38.4 \%$ en el grupo estudio. (Fig. 1). La segunda causa de cesárea lo ocupó el antecedente de cesárea ( $23.07 \%$ vs $46.1 \%$, controles vs. casos).

\section{Tabla 1}

OBESIDAD EMBARAZO Y DIETA Resultado Perinatal

\begin{tabular}{|c|c|c|c|}
\hline & Dieta $(\%)$ & No dieta $(\%)$ & Total (\%) \\
\hline Macrosómicos & $4 / 30(13.3)$ & $4 / 30(13.3)$ & $8 / 60(13.3)$ \\
\hline Cesáreas & $14 / 30(46.6)$ & $12 / 30(40.0)$ & $16 / 60(43.3)$ \\
\hline Apgar & $\geqslant 7(100)$ & $\geqslant 7(100)$ & \\
\hline E. Prolongado & & & $5 / 60(8.33)$ \\
\hline
\end{tabular}

La incidencia de macrosómicos, embarazos prolongados y el puntaje de Apgar fueron similares en ambos grupos. Los datos en paréntesis corresponden a porcentajes.

Figura 2

\section{OBESIDAD Y DIETA} PESO PROMEDIO DEL RECIEN NACIDO

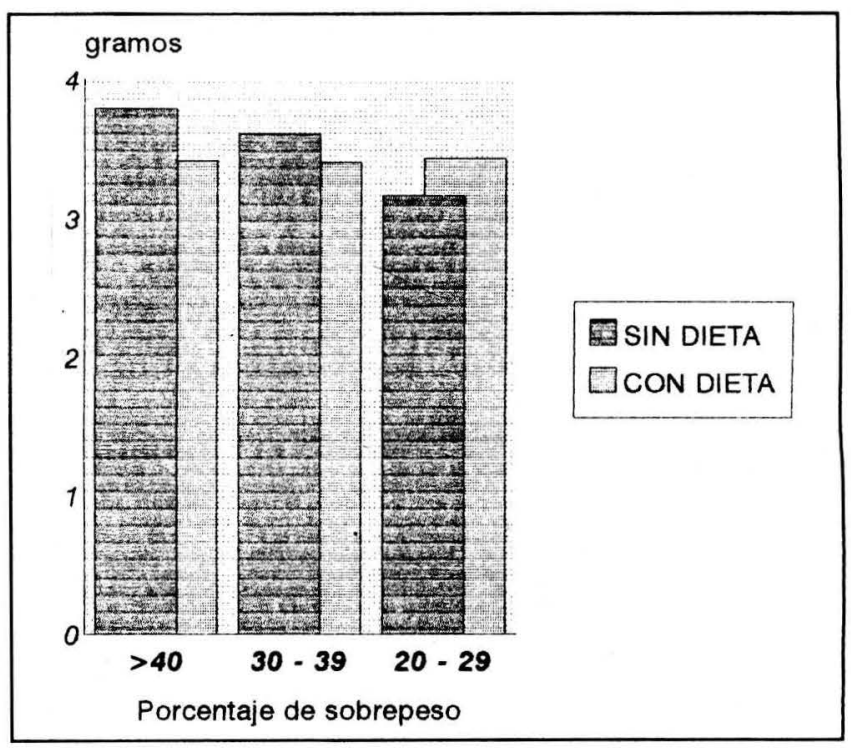

La diferencia de peso del $R N$ no es significante entre los grupos con dieta y los controles $(p=0.01)$ 
Figura 1

OBESIDAD EMBARAZO Y DIETA INCIDENCIA Y CAUSAS DE CESAREAS

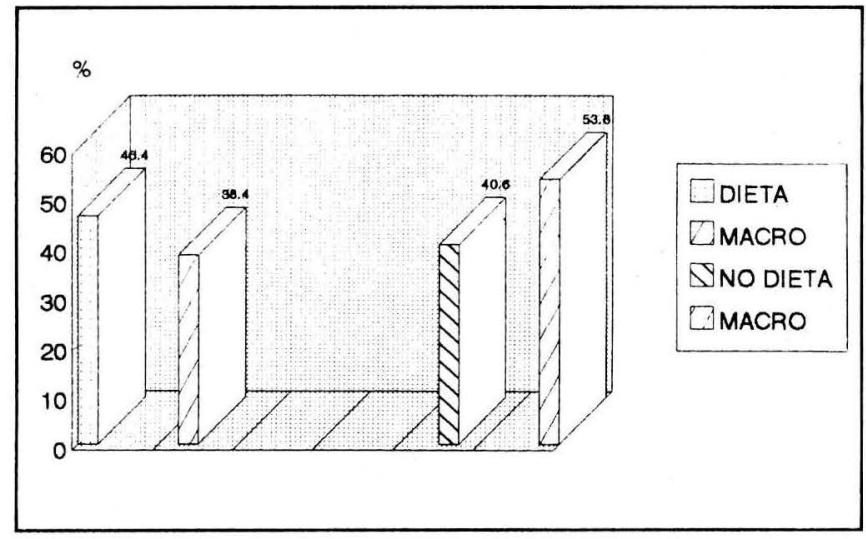

La incidencia de cesáreas por $R N$ de mayor peso para la edad gestacional, es significativamente mayor en los pacientes controles que no reciben dieta.

\section{Figura 3 \\ OBESIDAD Y EMBARAZO ANTECEDENTES FAMILIARES Y PERSONALES}

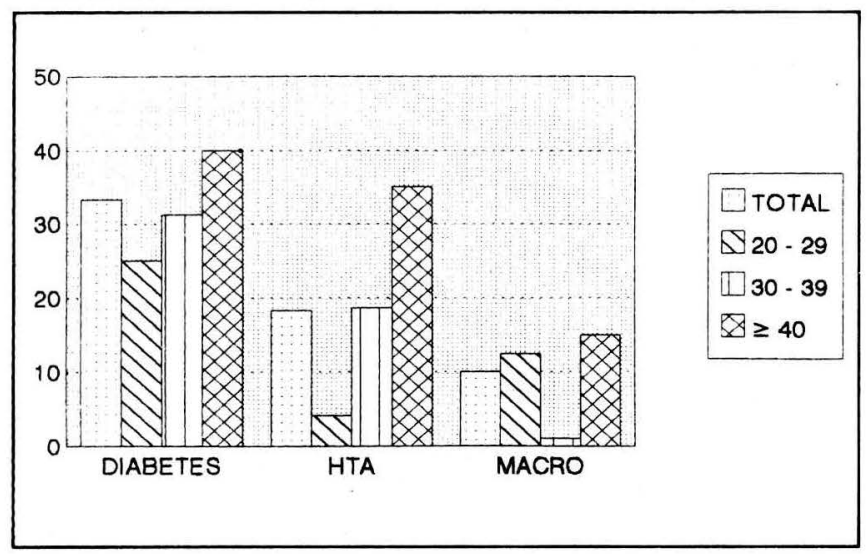

Los antecedentes familiares de Diabetes y familiares y personales de HTA, son más frecuentes en las pacientes con $\geqslant 40 \%$ de sobrepeso con relación al total de las pacientes obesas.

Cuando se analizan individualmente los grupos de acuerdo con el porcentaje de sobrepeso, se encuentra:

$340 \%$ de sobrepeso: a) las semanas de gestación entre los grupos SIN dieta y CON dieta son comparables (38.2 vs $38.2 ; \mathrm{p}=0.5$; b) no hay diferencia en el peso del RN en los grupos CON y SIN dieta; c) no hay correlación entre semanas de gestación y peso del RN en el grupo SIN dieta $(p=0.09)$ d) si hay correlación entre peso del RN y semanas de gestación entre el grupo CON dieta ( $\mathrm{p} \gg 0)$.

$30-39 \%$ de sobrepeso: a) No hay diferencia entre semanas de gestación en los dos grupos; b) tampoco hay diferencia en los pesos del RN; c) no hay correlación entre peso del RN y semanas de gestación en el grupo SIN dieta $(\mathrm{p}=0.5)$; $\mathrm{d})$ la correlación en el grupo CON dieta alcanza un $\mathrm{p}=0.08$, que no es significativo.
20-29\% de soprepeso: a) semanas de gestación y peso del RN no son diferentes; b) no hay correlación entre peso del RN vs. semanas de gestación en grupo SIN dieta $(p=0.28) ; c)$ si hay correlación entre semanas de gestación y peso del RN en grupo CON dieta $(p=0.02)$.

Es notable el dato de 20 pacientes de las 60 pacientes de ambos grupos (33.3\%) tenían antecedentes familiares de diabetes. Relacionado con el porcentaje de sobrepeso, cuando mayor éste se encuentra con mayor frecuencia el antecedente ( ${ }^{3}$ 40: 40\%; 30-39: 31.2\%; 20-29: 25\%) Fig. 3.

La patología agregada fue primariamente hipertensión arterial en el $12.06 \%$ y toxemia en el $10.34 \%$.

\section{Discusión}

El embarazo es considerado un estado diabetogénico, atribuible a la presencia de niveles altos de hormonas como lactógeno placentario, prolactina, progesterona, estrógenos y cortisol entre los más importantes (1).

El objetivo de la dieta en la diabética gestacional es controlar la hiperglicemia y prevenir la cetonemia, permitiendo una ganancia de peso compatible con un adecuado crecimiento fetal (10).

La paciente obesa debe tener una ganancia de peso que permita un desarrollo fetal adecuado $(9-12 \mathrm{~kg}$. en promedio), proscribiéndose las dietas reductoras de peso por la deprivación calórica al feto y la subsecuente cetonemia materno-fetal (17). Una dieta similar a la usada en las diabéticas gestacionales con una restricción calórica máxima del $33 \%$.

(1.600-1.800 calorías) es más apropiada y puede ser más efectiva que la administración de insulina (11).

La obesidad ha sido relacionada con alteraciones metabólicas que constituyen fuente de variada morbilidad (12).

La más constante asociación es con diabetes mellitus. Se reconoce que la mayoría de los pacientes diabéticos no insulino dependientes son obesos.

Es notable en este estudio una relación entre el peso incrementado del RN y la mayor frecuencia de antecedentes familiares de diabetes.

Por otra parte la obesidad como tal se considera en la clasificación de diabetes (13).

Un hallazgo constante en pacientes obesos es la hiperinsulinemia basal y la inducida por administración de glucosa, aminoácidos, glucagón y tolbutamida. Existe también en estos pacientes resistencia a la insulina que no los hace propensos a la hipoglicemia.

La hiperinsulinemia a su vez, es debida al aumento de producción de insulina por un páncreas con células beta hipertrofiadas y una disminución de la depuración de insulina por el hígado (1).

El embarazo se acompaña de cambios en el metabolismo de la glucosa, atribuibles a la producción de hormonas placentaria como el HPL, estrógenos, progesterona, cortisol y' sustancias como el colesterol, que ejercen efectos periféricos de resistencia a la insulina, dependientes de un mecanismo postreceptor (1).

La obesidad ha sido relacionada con factores de riesgo maternos $y$ perinatales. La macrosomia fetal ha 
sido reportada por varios autores en las pacientes obesas embarazadas $(3-4,12-14)$.

Aunque no se ha definido la razón por la cual la obesidad promueve el desarrollo de niños de peso mayor que los no obesos, la hiperinsulinemia materna relacionada con la obesidad puede ser el mecanismo disparador (15).

De todas maneras la morbilidad materna y fetal está relacionada con el sobrepeso fetal: fractura de clavícula, parálisis del plexo braquial, parálisis facial, hipoglicemia y sufrimiento fetal; hemorragia, trauma perineal, infecciones y hematomas de la episiotomia por el lado materno (12).

La incidencia del 5\% de macrosómicos en la población embarazada general en nuestro medio se ve incrementada a un $13.3 \%$ en las pacientes obesas en este estudio.

En el presente estudio se comparó un grupo de obesas tratadas con la dieta usada para el manejo de la diabética gestacional, y no se encontró diferencia en la morbimortalidad fetal comparada con el grupo que no recibió dieta.

Aunque el peso promedio de los recién nacidos de pacientes obesas con dieta y sin dieta fue sensiblemente igual, se encontró un dato interesante y fue la correlación entre la edad gestacional y el peso del RN en las pacientes con dieta, contrario a lo que pasa con las que no reciben dieta. Esto lo hemos analizado como una posible regulación positiva en el metabolismo energético madre feto, en las pacientes que reciben dieta, que podría manifestar- se como una adecuada regulación metabólica intrauterina, reflejada en la evolución normal del crecimiento ponderal fetal y por ende, en la época prenatal, con bienestar metabólico fetal, que puede incidir en el resto de vida del individuo (15).

Aunque la frecuencia de cesáreas en el grupo estudio (46.4), es similar al del grupo control (40.6), en el 53.8\% de las pacientes que terminaron el embarazo por intervención quirúrgica en este último grado la causa fue el alto peso fetal para la edad gestacional. El ligeramente mayor número de cesáreas en el grupo estudio vs. el control, se explica por un mayor antecedente de cesáreas previas en ese grupo de pacientes, posiblemente, aunque no lo investigamos, por desproporción feto-pélvica en embarazos anteriores. Aunque como se dijo, no se registró una mayor morbilidad fetal y el peso del RN fue sensiblemente igual en ambos grupos con y sin dieta, en el grupo estudio, similar a lo encontrado en el manejo de la diabética gestacional, en la cual una moderada restricción de la ingesta calórica, muestra tendencia a la disminución de glucosa y triglicéridos, sugiriendo que es de apreciable valor en la prevención de recién nacidos de peso alto para la edad gestacional de macrosómicos (16), o por lo menos, de regular el metabolismo energético fetal para un desarrollo adecuado (16).

Por lo tanto creemos que la paciente obesa debe manejarse como una paciente diabética gestacional tanto desde el punto de vista metabólico, como de monitoría del embarazo.

\section{BIBLIOGRAFIA}

1. Glass A. Endocrine aspects of obesity. Medical Clinics of North America 1989; 73: 139.

2. Gray D. Diagnosis and prevalence of obesity. Med. Clin. North. Am. 1989; 73: 1 .

3. Colandra C., Abel DA., Busher NA. Maternal obesity in pregnancy. Obstet. Gynecol. 1981; 57: 8.

4. Gross T., Sokol RJ., King KC. Obesity in pregnancy. Risk and outcome. Obstet. Gynecol. 1980; 56: 446.

5. Edwards LE., Dickes WF., Alton IR. et al. Pregnancy in the massively obese: cause, outcome and obesity prognosis of the infant. Am. J. Obstet. Gynecol. 1978; 131: 479.

6. Gómez G., Orjuela JL., Moreno J. Obesidad y embarazo. Rev. Col. Obstet. Ginecol. 1993; 44: 283.

7. Manson JE., Stamfer MJ., Hennekens CH. Body weight and longevity: a reassessment. JAMA 1987; 256: 353.

8. Azziz R. Reproductive endocrinologic alterations in female asymptomatic obesity. Fertil. Steril. 1989; 52: 703.

9. Gómez G. Diabetes y embarazo. Protocolo de manejo. Rev. Col. Obstet. Gynecol. 1990; 41: 81 .
10. Mann JL. Diet and diabetes. Diabetología 1980; 18: 89 .

11. Nelson M. Diabetes and pregnancy. In high-risk pregnancy and delivery. Arias F. (ed.) Mosby Co. 1984; 132.

12. Ruge S., Anderson T. Obstetrics risk in obesity. An analysis of the literature. Obstet. Gynecol. Surv. 1985; 40: 57.

13. National Diabetes Data Group: Clasification and diagnosis of diabetes mellitus and others categories of glucose intolerance. Diabetes 1979; 28: 1039.

14. Panel P., de Meeus JB., Yanoulopoulos B., Deshayes M., Magnin G. Delivery of large infants. Management and result of 198 cases. J. Gynecol. Obstet. Biol. Reprod. (Paris) 1991; 20: 729.

15. Kalkhoff RK. Impact of maternal fuels and nutritional state on fetal growth. Diabetes 1991; 40(2): 61.

16. Knopp mRh., Magee MS., Raisys V., Benedetti T. Metabolic effects of hypocaloric diets in management of gestational diabetes. Diabetes 1991; 40(2): 165. 